\title{
Erratum
}

\section{Galaxy clusters in the CFHTLS}

\section{First matched filter candidate catalogue of the Deep fields}

\author{
L. F. Olsen ${ }^{1,2, \star}$, C. Benoist ${ }^{2}$, A. Cappi ${ }^{2,3}$, S. Maurogordato ${ }^{2}$, A. Mazure ${ }^{4}$, E. Slezak ${ }^{2}$, C. Adami ${ }^{4}$,
} C. Ferrari ${ }^{5}$, and F. Martel $^{2}$

\footnotetext{
1 Dark Cosmology Centre, Niels Bohr Institute, University of Copenhagen, Juliane Maries Vej 30, 2100 Copenhagen, Denmark e-mail: lisbeth@dark-cosmology.dk

2 Observatoire de la Côte d'Azur, Laboratoire Cassiopée, BP 4229, 06304 Nice Cedex 4, France

3 INAF - Osservatorio Astronomico di Bologna, Via Ranzani 1, 40127 Bologna, Italy

${ }^{4}$ Laboratoire d'Astrophysique de Marseille, UMR 6110 CNRS-Université de Provence, BP 8, 13376 Marseille Cedex 12, France

${ }^{5}$ Institut für Astro- und Teilchen Physik, Universität Innsbruck, Technikerstraße 25, 6020 Innsbruck, Austria
}

A\&A 461, 81-93 (2007), DOI:10.1051/0004-6361:20066088

\begin{abstract}
Key words. methods: data analysis - surveys - galaxies: clusters: general - cosmology: large-scale structure of Universe -
\end{abstract} errata, addenda

Table 1. Corrected table.

\begin{tabular}{ll}
\hline \hline Parameter & Value \\
\hline \multicolumn{2}{c}{ Filter } \\
Core radius, $r_{\mathrm{c}}$ & $0.1 h_{75}^{-1} \mathrm{Mpc}$ \\
Cut off radius, $r_{\mathrm{co}}$ & $1.0 h_{75}^{-1} \mathrm{Mpc}$ \\
\hline
\end{tabular}

After publication of Olsen et al. (2007) we noted that there was a mistake in Table 1 where the first two lines are corrected in Table 1 of the present erratum to reflect the actual parameter settings for building the catalogue.

We also noted that in our peak detection we used a wrong detection threshold, too high by a factor of 10 , for the highest redshift bin. We have, therefore, reextracted cluster catalogues and give here the list of missing candidates in Table 3. All the missed candidates are at $z=1.2$. For the already published candidates the parameters are not changed by this correction.

With these additional candidates the total number of cluster candidates is increased to 169 corresponding to a density of $54.4 \pm 7.0$ per square degree. With these new settings the number of false detections is increased to $16.9 \pm 5.4$ per square degree. The updated Fig. 11 gives the redshift and richness distributions of the real and false detections.

We also provide an updated version of the recovery rate in Fig. 5. It can be seen that only minor changes occur at the highest redshifts.
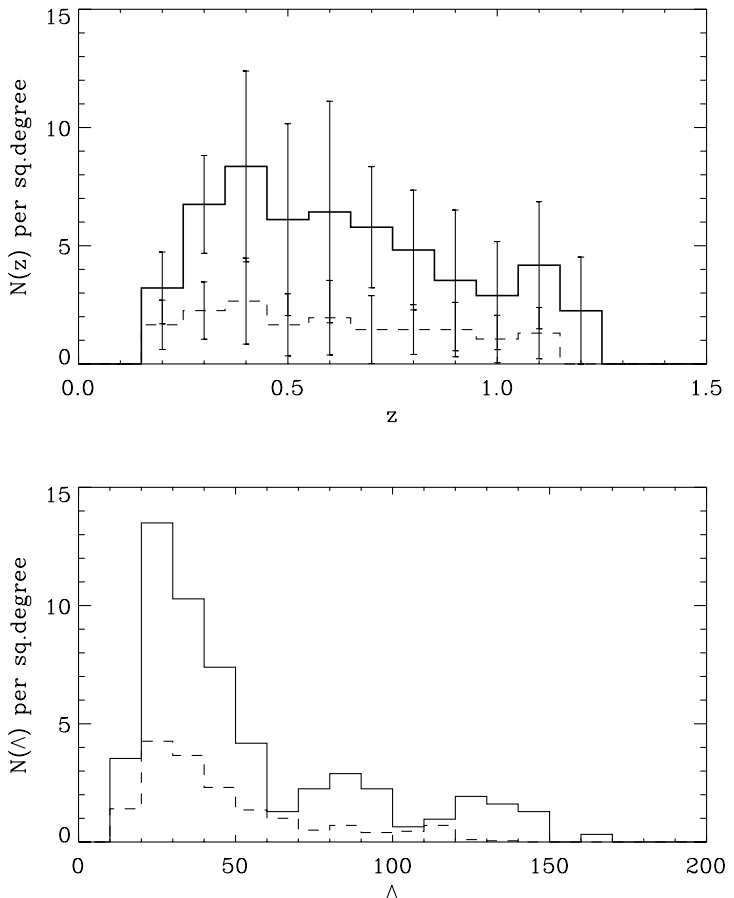

Fig. 11. Redshift (top) and richness (lower) distributions (solid lines) for all the candidate clusters. The distributions of false detections (dashed lines) are estimated using the correlated backgrounds. For the redshift distribution the error bars denote the scatter between the fields. Corresponding to Fig. 11 in the original paper.

^ Now L. F. Grove. 
Table 3. Cluster candidates missing in the original publication.

\begin{tabular}{|c|c|c|c|c|c|c|c|c|}
\hline Name & $\overline{\alpha \alpha(\mathrm{J} 2000)}$ & $\overline{\delta \delta(\mathrm{J} 2000)}$ & 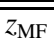 & 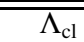 & 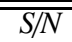 & \# Bins & Frac. of lost area & $\overline{\text { Grade }}$ \\
\hline CFHTLS-CL-J022427-040928 & $02: 24: 27.0$ & $-04: 09: 28.1$ & 1.2 & 135.3 & 3.74 & 2 & 0.00 & $\mathrm{C}$ \\
\hline CFHTLS-CL-J022501-041329 & $02: 25: 01.0$ & $-04: 13: 29.4$ & 1.2 & 149.7 & 4.14 & 2 & 0.00 & $\mathrm{C}$ \\
\hline CFHTLS-CL-J100150+020950 & 10:01:50.7 & $+02: 09: 50.7$ & 1.2 & 145.9 & 3.69 & 2 & 0.00 & $\mathrm{C}$ \\
\hline CFHTLS-CL-J221418-174507 & $22: 14: 18.9$ & $-17: 45: 07.8$ & 1.2 & 143.6 & 4.10 & 2 & 0.00 & $\mathrm{C}$ \\
\hline CFHTLS-CL-J221502-175611 & $22: 15: 02.8$ & $-17: 56: 11.7$ & 1.2 & 126.3 & 3.62 & 2 & 0.00 & $\mathrm{D}$ \\
\hline CFHTLS-CL-J221558-173757 & $22: 15: 58.9$ & $-17: 37: 57.9$ & 1.2 & 134.8 & 3.86 & 2 & 0.00 & A \\
\hline CFHTLS-CL-J221700-180957 & $22: 17: 00.6$ & $-18: 09: 57.2$ & 1.2 & 129.2 & 3.71 & 2 & 0.00 & $\mathrm{C}$ \\
\hline
\end{tabular}

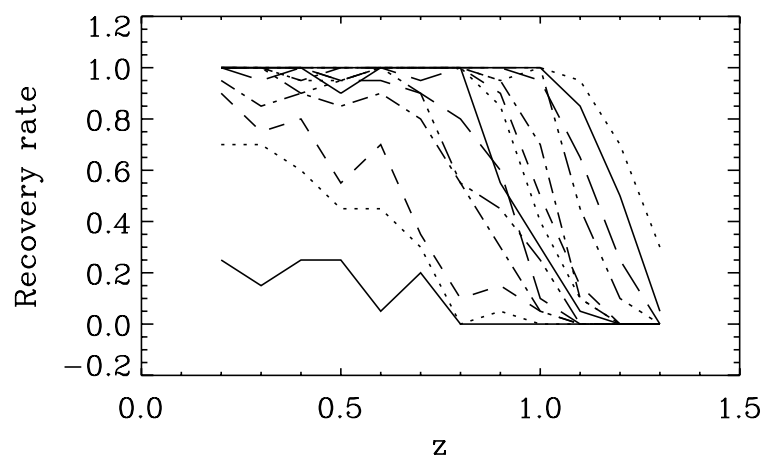

Fig. 5. The detection efficiency for the correlated background $\sigma_{\text {det }}=$ $3.5 \sigma$, area $\sim \pi\left(0.5 \mathrm{r}_{\mathrm{c}}\right)^{2}$. The lines correspond to $\Lambda_{\mathrm{cl}}=10-300$ from left to right. 